ESAIM: PROCEEDINGS AND SURVEYS, October 2015, Vol. 51, p. 175-192

A. Garivier et al, Editors

\title{
STOCHASTIC GEOMETRY: BOOLEAN MODEL AND RANDOM GEOMETRIC GRAPHS
}

\author{
Pierre Calka $^{1}$, David Coupier ${ }^{2}$, Jean-Baptiste Gouéré $^{3}$, Arnaud Rousselle $^{4}$ \\ AND ANAÏS VERGNE ${ }^{5}$
}

\begin{abstract}
This paper collects the four contributions which were presented during the session devoted to Stochastic Geometry at the journées MAS 2014. It is focused in particular on several questions related to the transmission of information in a general sense in different random media. The underlying models include the Boolean model, simplicial complexes or geometric random graphs induced by a point process.

Résumé. Cet article propose les quatre contributions présentées lors de la session Géométrie stochastique des journées MAS 2014. Il est centré en particulier sur plusieurs questions de transmission de l'information au sens très général dans différents milieux aléatoires. Les modèles sous-jacents sont notamment le modèle Booléen, des complexes simpliciaux ou des graphes géométriques aléatoires engendrés par un processus ponctuel.
\end{abstract}

\section{INTRODUCTION}

Stochastic geometry is a branch of probability theory which consists in studying random spatial models embedded in the Euclidean space $\mathbb{R}^{d}, d \geq 2$, or any metric space. The roots of stochastic geometry go back to well-known questions from recreational mathematics like the Buffon's needle problem (1733). Notably because of the need for satisfying spatial models in experimental science (geology, material physics, cellular biology...), it has emerged in the second half of the twentieth century as a new domain at the intersection of probability theory, Euclidean geometry and integral geometry. The expression Stochastic Geometry was notoriously used for the first time by D. G. Kendall and K. Krickeberg during an Oberwolfach conference in 1969.

The models from stochastic geometry can be for instance point processes, i.e. random locally finite subsets or they can be geometric constructions induced by point processes such as unions of balls, random tessellations, random polytopes or random graphs. Particular emphasis is given here to recent discoveries on both the Boolean model and several types of random geometric graphs. A well-known application field of these two kinds of models is the study of telecommunication networks and questions related to coverage optimization. To some extent, the four contributions below share a common approach: they describe a random environment where the randomness is due essentially to the underlying point process and they investigate how the transmission of information behaves inside this random environment.

${ }^{1}$ LMRS, université de Rouen, avenue de l'université, BP12, 76801 Saint-Étienne-du-Rouvray, France

${ }^{2}$ Laboratoire Paul Painlevé, Université Lille 1, Cité scientifique, 59655 Villeneuve d'Ascq Cedex, France

3 MAPMO, université d'Orléans, BP 6759, 45067 Orléans Cedex 2, France

${ }^{4}$ LMRS, université de Rouen, avenue de l'université, BP12, 76801 Saint-Étienne-du-Rouvray, France

${ }^{5}$ INRIA Saclay Ile-de-France, 1 rue Honoré d'Estienne d'Orves Campus de l'École Polytechnique 91120 Palaiseau, France

(C) EDP Sciences, SMAI 2015 
The Boolean model in its basic form is a random covering of $\mathbb{R}^{d}$ by the union of random balls centered at the points of a point process. Classical questions include continuum percolation, i.e. the existence of an unbounded connected component of the occupied or vacant phase. On a side note, the Boolean model gives birth to a random geometric graph where the set of vertices is the point process itself and there is an edge between two vertices if their corresponding balls intersect. In the contribution of Jean-Baptiste Gouéré, the central question is related to first-passage percolation: assuming that the speed is infinite in the occupied phase and equal to 1 in the vacant phase, what is the minimal amount of time needed to go from $x$ to $y, x$ and $y$ being two fixed points of $\mathbb{R}^{d}$ ?

A particular Boolean model where all the balls have the same deterministic radius is also the starting point of Anaïs Vergne's work. For a given point process and a fixed radius $r>0$, the random geometric graph described above is constructed and more generally, the topological properties of the union of balls are described via combinatorial objects called simplicial complexes. The aim is here to define a strategy for recovering a damaged cellular network. The first two Betti numbers indicate whether it is repaired or not.

The contribution of David Coupier shares with Anaïs Vergne's effort the construction via some precise geometric rules of random graphs which have a point process as vertex set. Moreover, it shares with JeanBaptiste Gouéré's work some concerns about first-passage percolation. It focuses on two classical examples of random geometric trees, namely the Radial Poisson Tree and the Euclidean First-Passage Percolation Tree and investigates the growth of the mean number of semi-infinite branches in these two trees.

Finally, Arnaud Rousselle introduces a different model of random geometric graph, i.e. the Delaunay triangulation induced by a point process. He is interested in the behavior of random walks in the random environment provided by such graph and more particularly in determining recurrence or transience criteria and showing an invariance principle.

The paper is structured as follows.

In the second section, Jean-Baptiste Gouéré deals with the Boolean model, i.e. the union of balls with random radius and centered at each point of a homogeneous Poisson point process of $\mathbb{R}^{d}$. A traveler moves with speed 1 outside of the Boolean model and with infinite speed inside the covered part. The minimal amount of time which is required to go from a point $a$ to a point $b$ far enough is close to $\mu\|b-a\|$ for some constant $\mu$. The aim is to find a lower bound for $\mu$. In order to do this, a continuous analogue of greedy paths is introduced and studied. This is joint work with Régine Marchand (Institut Élie Cartan, University of Lorraine, France) which is motivated by a previous model due to Deijfen.

In the third section, Anaïs Vergne considers a damaged cellular network, presenting disconnected components and/or coverage holes. She proposes a recovery algorithm that provides the locations where the network operator needs to add nodes to patch the existing network. The use of the simplicial complex representation for wireless networks allows to compute the connectivity and the coverage of the network. Then the algorithm adds new nodes in excessive number following a given method. Finally a reduction algorithm under topology invariance constraint is run on the added nodes in order to reach a maximal result. The addition method in particular uses the Ginibre point process which induces repulsion between points. This is joint work with Ian Flint (Nanyang Technology University, Singapore), Laurent Decreusefond and Philippe Martins (Institut Telecom, TELECOM ParisTech, LTCI, Paris, France).

It is known that the number $\chi_{r}$ of semi-infinite branches crossing the sphere $S(O, r)$ in a straight geometric random tree almost surely tends to infinity with $r$. In the fourth section, David Coupier develops a general method ensuring the expectation of $\chi_{r}$ is sublinear and he applies it to two different trees, namely the Radial Poisson Tree and the Euclidean First-Passage Percolation Tree.

In the fifth and final section, Arnaud Rousselle considers simple random walks on Delaunay triangulations generated by point processes in the Euclidean space $\mathbb{R}^{d}$. Under suitable assumptions on the point processes, he shows that for almost any realization of the point process, these random walks are recurrent if $d=2$ and transient if $d \geq 3$. Moreover, the random walk satisfies an almost sure (or quenched) invariance principle. This holds for point processes which have clustering or repulsiveness properties including Poisson point processes, 
Matérn cluster and Matérn hardcore processes. The method relies in particular on the decomposition of the process into a martingale part and a corrector which is proved to be negligible at the diffusive scale

\section{BoOleAn MODEL, FIRST-PASSAge PERCOLATION AND GREEDy PATHS}

\subsection{Model and results}

\subsubsection{Boolean model}

The Boolean model is defined as follows. At each point of a homogeneous Poisson point process on the Euclidean space $\mathbb{R}^{d}$, we center a ball of random radius. We assume that the radii of the balls are independent, identically distributed and independent of the point process. The Boolean model is the union of the balls. Three parameters are involved:

- $\lambda$ : the intensity measure of the Poisson point process is $\lambda$ times the Lebesgue measure on $\mathbb{R}^{d}$.

- $R$ : the radii are copies of the random variable $R$.

- $d$ : the action takes place in $\mathbb{R}^{d}$.

We will denote the Boolean model by $\Sigma$ or by $\Sigma(\lambda, R, d)$. We refer to the book by Meester and Roy 37 for background on the Boolean model.

\subsubsection{Percolation in the Boolean model}

Consider the following event: one of the connected components of $\Sigma$ is unbounded. By ergodicity, the probability of this event is either 0 or 1 . In the first case one says that $\Sigma$ does not percolate. In the second case, one says that $\Sigma$ percolates. We define a critical threshold as follows:

$$
\lambda_{c}=\lambda_{c}(R, d)=\inf \{\lambda>0: \Sigma(\lambda, R, d) \text { percolates }\} \in[0,+\infty] .
$$

Two cases occur.

- If $\mathbb{E}\left(R^{d}\right)$ is infinite, then for all $\lambda>0$, with probability $1, \Sigma=\mathbb{R}^{d}$. The model is therefore trivial from the percolation point of view and one has $\lambda_{c}(R, d)=0$.

- If $\mathbb{E}\left(R^{d}\right)$ is finite, then $0<\lambda_{c}(R, d)<+\infty$.

In the previous statement, the difficult part is to prove that $\lambda_{c}(R, d)$ is positive when $\mathbb{E}\left(R^{d}\right)$ is finite. The question was raised by Hall in [27] and solved by the author in [21].

In what follows we always assume that $\mathbb{E}\left(R^{d}\right)$ is finite. We refer to the book by Grimmett [26] for background on discrete percolation and to the book by Meester and Roy [37] for background on percolation in the Boolean model.

\subsubsection{First-passage percolation in the Boolean model}

In [22], Régine Marchand and the author studied a model introduced by Deijfen in [13]. The model we introduce in this section appears implicitly in $[22$ as an intermediate model. We refer to 22 for the definition of Deijfen's model and its links with the model defined here.

A traveler walks on $\mathbb{R}^{d}$. Inside the Boolean model $\Sigma$ he walks at infinite speed. Outside the Boolean model $\Sigma$ he walks at speed 1 . He travels from $x \in \mathbb{R}^{d}$ to $y \in \mathbb{R}^{d}$ as fast as he can. We denote by $T(x, y)$ the time needed to perform this travel. For example if $x$ and $y$ belong to the same connected component of $\Sigma$, then $T(x, y)=0$.

Here is a more formal definition. For any $a$ and $b$ in $\mathbb{R}^{d}$, we define $\tau(x, y)$ as the 1-dimensional Lebesgue measure of $[a, b] \backslash \Sigma$. A path is a finite sequence of distinct points of $\mathbb{R}^{d}$. To each path $\pi=\left(x_{0}, \ldots, x_{n}\right)$ is associated a time as follows:

$$
\tau(\pi)=\sum_{i=1}^{n} \tau\left(x_{i-1}, x_{i}\right) .
$$

If $x$ and $y$ are two points of $\mathbb{R}^{d}$, then $T(x, y)$ is defined by:

$$
T(x, y)=\inf \{\tau(\pi): \pi \in \mathcal{C}(x, y)\}
$$


where $\mathcal{C}(x, y)$ is the set of paths from $x$ to $y$.

A standard application of Kingman sub-additive theorem yields the following result. There exists a constant $\Theta=\Theta(\lambda, R, d) \in[0,1]$ such that:

$$
\frac{T(0, x)}{\|x\|} \rightarrow \Theta \text { with probability } 1 \text { and in } L^{1} \text { as }\|x\| \rightarrow \infty .
$$

\subsubsection{Positivity of $\Theta$}

Let us begin by two simple remarks.

- If $\lambda>\lambda_{c}$, then with probability 1 , one of the connected component of $\Sigma$ is unbounded. As the speed is infinite inside $\Sigma$, one easily gets $\Theta=0$.

- If $\lambda<\lambda_{c}$, then with probability 1 , all the connected components of $\Sigma$ are bounded. One can check that this implies the following convergence:

$$
T(0, x) \rightarrow \infty \text { as }\|x\| \rightarrow \infty .
$$

The previous result does not imply the positivity of $\Theta$. Without further assumptions the positivity of $\Theta$ is an open problem. If $\Theta$ is bounded one can adapt the proof of Kesten in the discrete setting [31] to prove the positivity of $\Theta$.

Let us consider the following threshold:

$$
\lambda_{\mu}=\lambda_{\mu}(R, d)=\inf \{\lambda>0: \Theta(\lambda, R, D)=0\} .
$$

One can sum up the previous remarks as follows:

- The inequality $\lambda_{\mu} \leq \lambda_{c}$ holds.

- Does the equality $\lambda_{\mu}=\lambda_{c}$ hold ?

Because of the link with Deijfen's model, we sought in 22$]$ a necessary and sufficient condition for the positivity of $\lambda_{\mu}$. Proving in full generality the equality $\lambda_{\mu}=\lambda_{c}$ would have provided such a condition. Indeed, $\lambda_{c}$ is positive if and only if $\mathbb{E}\left(R^{d}\right)$ is finite. However we failed at proving in full generality the equality $\lambda_{\mu}=\lambda_{c}$.

We therefore used another strategy and proved the following result (the result is implicit in $[22]$ ).

Theorem 1. (1) If

$$
\int_{0}^{+\infty} \mathbb{P}(R \geq r)^{1 / d} d r<\infty
$$

then $\lambda_{\mu}(R, d)$ is positive.

(2) If $\lambda_{\mu}(R, d)$ is positive, then

$$
\mathbb{E}\left(R^{d}\right)<\infty
$$

Note that (1) holds as soon as there exists $\epsilon>0$ such that:

$$
\mathbb{E}\left(R^{d}\left(\ln (R)_{+}\right)^{d-1+\epsilon}\right)<+\infty
$$

The result is straightforward when $d=1$. The second item is also straightforward in any dimension. The proof of the first item when $d \geq 2$ relies on the model of greedy paths that we now introduce.

\subsubsection{Greedy paths in the Boolean model}

Denote by $\mathcal{N}$ the set of centers of the balls. This is a homogeneous Poisson point process. With each $c \in \mathcal{N}$ is associated a radius that we denote by $R(c)$. If $c \in \mathbb{R}^{d}$ does not belong to $\mathcal{N}$, we set $R(c)=0$. 
As before a path is a finite sequence $\pi=\left(x_{0}, \cdots, x_{n}\right)$ of distinct points of $\mathbb{R}^{d}$. We denote by $\ell(\pi)$ the length of the path:

$$
\ell(\pi)=\sum_{i=1}^{n}\left\|x_{i}-x_{i-1}\right\|
$$

We also define a weight $R(\pi)$ as follows:

$$
R(\pi)=\sum_{i=0}^{n} R\left(x_{i}\right)
$$

We are interested in the following quantity:

$$
S=\sup \left\{\frac{R(\pi)}{\ell(\pi)}\right\}
$$

where the supremum is taken over any path of any positive length originating from 0 . In [22, we prove the following result:

Theorem 2. Assume $d \geq 2$.

(1) If (1) holds, then $\mathbb{E}(S)$ is finite.

(2) If $\mathbb{E}(S)$ is finite, then (2) holds.

This is the analogue in the continuous setting of a result of Martin 34] in the discrete setting. The proof is actually more natural in the continuous setting. The second item is straightforward.

\subsection{Some proofs}

\subsubsection{Idea of the proof of Item 1 of Theorem 2}

Let us write $S(\lambda, R, d)$ to emphasize the dependance on the parameters of the Boolean model. The key is the following scaling relation. For any $\lambda, R, d$ :

$$
S(\lambda, R, d) \stackrel{\text { law }}{=} \lambda^{1 / d} S(1, R, d) .
$$

This can be seen by applying an homothetic transformation with ratio $\lambda^{1 / d}$ to the set of centers of $\Sigma(\lambda, R, d)$.

Then, we can proceed for example as follows. For all path $\pi=\left(x_{0}, \ldots, x_{n}\right)$ we have:

$$
\frac{R(\pi)}{\ell(\pi)}=\int_{0}^{+\infty} \frac{\sum_{i=0}^{n} 1_{r \leq R\left(x_{i}\right)}}{\ell(\pi)} d r
$$

Therefore:

$$
\begin{aligned}
S(\lambda, R, d) & =\sup _{\pi} \int_{0}^{+\infty} \frac{\sum_{i=0}^{n} 1_{r \leq R\left(x_{i}\right)}}{\ell(\pi)} d r \\
& \leq \int_{0}^{+\infty} \sup _{\pi} \frac{\sum_{i=0}^{n} 1_{r \leq R\left(x_{i}\right)}}{\ell(\pi)} d r
\end{aligned}
$$

Note:

Taking expectation and using the scaling we then get:

$$
\sup _{\pi} \frac{\sum_{i=0}^{n} 1_{r \leq R\left(x_{i}\right)}}{\ell(\pi)} \stackrel{\operatorname{law}}{=} S(\lambda \mathbb{P}(r \leq R), 1, d) .
$$

$$
\mathbb{E}(S(\lambda, R, d)) \leq \lambda^{1 / d} \int_{0}^{+\infty} \mathbb{P}(R \geq r)^{1 / d} d r \mathbb{E}(S(1,1, d)) .
$$

It suffices to check that $\mathbb{E}(S(1,1, d))$ is finite (this is the only place where we use $d \geq 2$ ) to complete the proof. 


\subsubsection{Idea of the proof of Item 1 of Theorem 1}

Let $x \in \mathbb{R}^{d}$. One seeks a lower bound for:

$$
T(0, x)=\inf \{\tau(\pi): \pi \in \mathcal{C}(0, x)\}
$$

In the infimum, one can restrict oneself to paths such that each segment satisfies one of the following conditions:

- One of the extremity of the segment is the center of a ball of $\Sigma$ and the segment is included in the ball.

- The segment does not touch any ball of $\Sigma$ (but its ends do).

For such a path $\pi=\left(x_{0}, \ldots, x_{n}\right)$ one has:

$$
\tau(\pi) \geq \ell(\pi)-2 R(\pi)
$$

Therefore:

$$
\frac{T(0, x)}{\|x\|} \geq \inf \left\{\frac{\tau(\pi)}{\ell(\pi)}\right\} \geq 1-2 \sup \left\{\frac{R(\pi)}{\ell(\pi)}\right\} \geq 1-2 S .
$$

Taking expectation and letting $\|x\|$ tend to infinity one gets:

$$
\Theta(\lambda, R, d) \geq 1-2 \mathbb{E}(S(\lambda, R, d))
$$

If (1) holds then $\mathbb{E}(S)$ is finite by Theorem 2 By scaling, we then get that $\Theta(\lambda, R, d)$ is positive for small enough $\lambda$. This completes the proof.

\section{Cellular network Recovery AFter A Disaster}

In case of a disaster, a cellular network can be seriously damaged: some of its nodes can be completely destroyed. Coverage holes can appear resulting in no signal for communication or no monitoring at all of a whole area, connectivity can be lost between nodes. Paradoxically, reliable and efficient communication is especially needed in such situations. Therefore solutions for damage recovery for the coverage of cellular networks are much needed. Extensive research on the coverage problem in wireless networks exists: we can cite location-based 15 and range-based [46] methods. However, connectivity based schemes seem of greater interest since they provide an exact mathematical description of coverage without any geographical (location or distance) information. In [24], the authors introduced the Vietoris-Rips complex, based on the proximity graph of a wireless network, as a tool to compute its topology. Coverage computation via simplicial homology comes down to linear algebra computations.

In this paper, we present a homology based algorithm for disaster recovery of cellular networks. We represent a network with the Cech simplicial complex characterizing its coverage. Our algorithm first adds supernumerary vertices in order to patch every existing coverage hole and connect every components, then runs an improved version of the reduction algorithm presented in [45] in order to reach a minimal result with a minimum number of added vertices. For the addition of new vertices, we compare classic methods to the use of a determinantal point process: the Ginibre point process. This process has the ability to create repulsion between vertices, and therefore has the inherent ability to locate areas with low density of vertices: namely coverage holes. We finally compare our whole disaster recovery algorithm performance to the classic recovery algorithm performance: the greedy algorithm for the set cover problem.

\subsection{Preliminaries}

We consider a damaged wireless network presenting coverage holes with a fixed boundary, possibly fictional but needed to define the domain to cover, of which we can see an example in Figure 1 . When representing a wireless network, a first idea will be a geometric graph, where sensors are represented by vertices and an edge is drawn whenever two sensors can communicate with each other. However, the graph representation has some limitations; first of all there is no notion of coverage. Graphs can be generalized to more generic combinatorial 


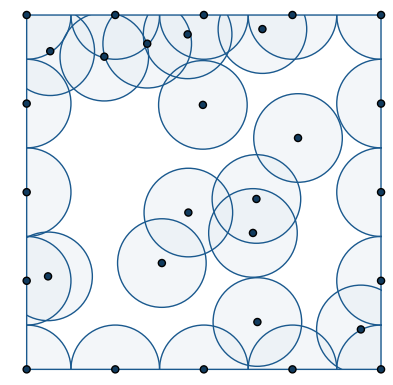

FiguRE 1. A damaged wireless network with a fixed boundary.

objects known as simplicial complexes. While graphs model binary relations, simplicial complexes represent higher order relations. A simplicial complex is a combinatorial object made up of vertices, edges, triangles, tetrahedra, and their $n$-dimensional counterparts. Given a set of vertices $V$ and an integer $k$, a $k$-simplex is an unordered subset of $k+1$ vertices $\left[v_{0}, v_{1} \ldots, v_{k}\right]$ where $v_{i} \in V$ and $v_{i} \neq v_{j}$ for all $i \neq j$. Thus, a 0 -simplex is a vertex, a 1-simplex an edge, a 2-simplex a triangle, a 3 -simplex a tetrahedron, etc.

Any subset of vertices included in the set of the $k+1$ vertices of a $k$-simplex is a face of this $k$-simplex. Thus, a $k$-simplex has exactly $k+1(k-1)$-faces, which are $(k-1)$-simplices. For example, a tetrahedron has four 3 -faces which are triangles. A simplicial complex is a collection of simplices which is closed with respect to the inclusion of faces, i.e. all faces of a simplex are in the set of simplices, and whenever two simplices intersect, they do so on a common face. An abstract simplicial complex is a purely combinatorial description of the geometric simplicial complex and therefore does not require the property of intersection of faces. For details about algebraic topology, we refer to $[28$. For a given set of vertices, and their coverage radii, we can build the Cech complex:

Definition 1 (C̆ech complex). Let $(\mathcal{X}, d)$ be a metric space where $\mathcal{X}$ is a set and $d$ a distance, $\omega$ be a finite set of $N$ points in $\mathcal{X}$, and $r$ be a real positive number. The Cech complex of $\omega$, denoted $\mathcal{C}_{r}(\omega)$, is the abstract simplicial complex whose $k$-simplices correspond to $(k+1)$-tuples of vertices in $\omega$ for which the intersection of the $(k+1)$ balls of radius $r$ centered at the $(k+1)$ vertices is non-empty.

The $k$-th Betti numbers of an abstract simplicial complex $\mathcal{X}$ are defined as the number of $k$-th dimensional holes in $\mathcal{X}$ and are computed via linear algebra computations. For example, $\beta_{0}$ counts the number of 0 dimensional holes, that is the number of connected components. And $\beta_{1}$ counts the number of holes in the plane. Therefore the Betti number $\beta_{1}$ of the Čch complex counts the number of coverage holes of the wireless network it represents.

\subsection{Vertices addition methods}

The algorithm begins by adding new vertices in addition to the set of existing vertices presenting coverage holes. For any non deterministic method, nodes are added until the network is repaired $\left(\beta_{0}=1\right.$ and $\left.\beta_{1}=0\right)$.

\subsubsection{Classic methods}

The first method we suggest is the grid method, it ensures perfect coverage: the new vertices are positioned along a square grid in a lattice graph where the distance between two neighboring vertices is $\sqrt{2} r$. The number of vertices is set. Therefore this method is completely independent from the initial configuration.

The second classic method is uniform positioning. The number of added vertices $N_{a}$ takes into account the number of existing vertices $N_{i}$ since we add nodes until the area is covered with both existing and added nodes. The new vertices are sampled following a uniform law on the entire domain.

We can see an example of the grid vertices addition method and the uniform positioning vertices addition on the damaged network of Figure 1 in Figure 2 New vertices appear in red. 

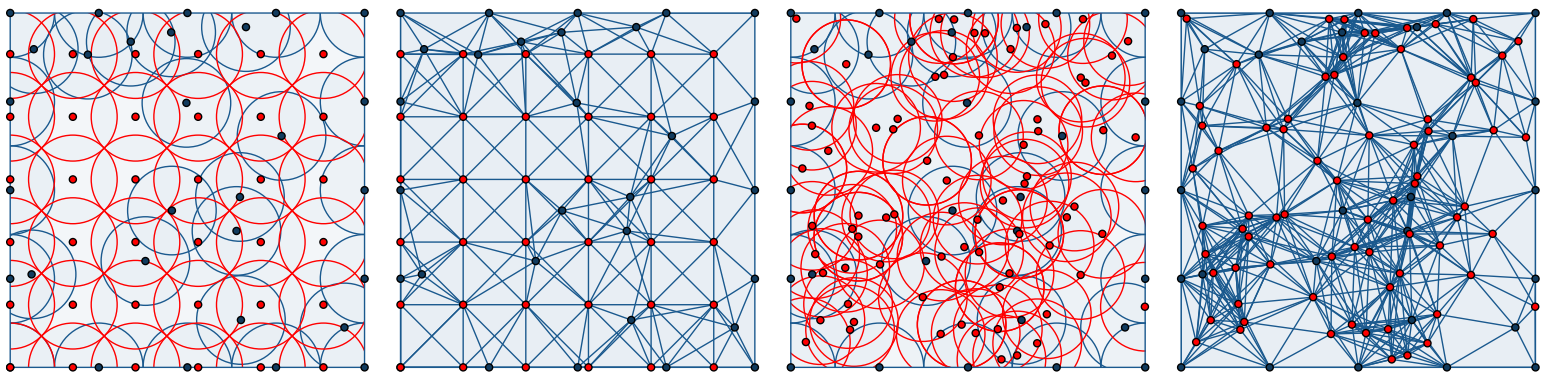

Figure 2. Classic addition methods (grid on left, uniform on right).

\subsubsection{Determinantal method}

In a Poisson point process, given the number of vertices, their positions are independent from each other. This independence can create aggregations of vertices, that is not convenient for our application. That is why we propose the use of processes creating some repulsion between vertices. General point processes can be characterized by their so-called Papangelou intensity $c$. Informally speaking, for $x$ a location, and $\omega$ a realization of a given point process, that is a set of vertices, $c(x, \omega)$ is the probability to have a vertex in an infinitesimal region around $x$ knowing the set of vertices $\omega$. For Poisson process, $c(x, \omega)=1$ for any $x$ and any $\omega$. A point process is said to be repulsive (resp. attractive) whenever $c(x, \omega) \geq c(x, \zeta)(\operatorname{resp} . c(x, \omega) \leq c(x, \zeta))$ as soon as $\omega \subset \zeta$. For repulsive point processes, that means that the greater the set of vertices is, the smaller the probability to have another vertex is. Among repulsive point processes, we are in particular interested in determinantal processes:

Definition 2 (Determinantal point process). Given $\mathcal{X}$ a Polish space equipped with the Radon measure $\mu$, and $K$ a measurable complex function on $\mathcal{X}^{2}$, we say that $\mathcal{N}$ is a determinantal point process on $\mathcal{X}$ with kernel $K$ if it is a point process on $\mathcal{X}$ with correlation functions $\rho_{n}\left(x_{1}, \ldots, x_{n}\right)=\operatorname{det}\left(K\left(x_{i}, x_{j}\right)_{1 \leq i, j \leq n}\right)$ for every $n \geq 1$ and $x_{1}, \ldots, x_{n} \in \mathcal{X}$.

We can see that when two vertices $x_{i}$ and $x_{j}$ tend to be close to each other for $i \neq j$, the determinant tends to zero for any kernel $K$ since two lines of the matrix tend to be similar. As a consequence, the correlation function tend to zero. That means that the vertices of $N$ repel each other. There exist as many determinantal point processes as functions $K$. We are interested in the following:

Definition 3 (Ginibre point process). The Ginibre point process is the determinantal point process with kernel $K(x, y)=\sum_{k=1}^{\infty} B_{k} \phi_{k}(x) \overline{\phi_{k}(y)}$, where $B_{k}, k=1,2, \ldots$, are $k$ independent Bernoulli variables and $\phi_{k}(x)=$ $\frac{1}{\sqrt{\pi k !}} e^{\frac{-|x|^{2}}{2}} x^{k}$ for $x \in \mathbb{C}$ and $k \in \mathbb{N}$.

The Ginibre point process is invariant with respect to translations and rotations, making it relatively easy to simulate on a compact set. Moreover, the repulsion induced by a Ginibre point process is of electrostatic type, creating circles of equal repulsion if there is only one vertex for example. Due to space limitations, we will not delve into the technicalities of the Ginibre point process simulation but they are developed in [12]. We can see a realisation of our simulation for the recovery of the wireless network of Figure 1 in Figure 3 . 

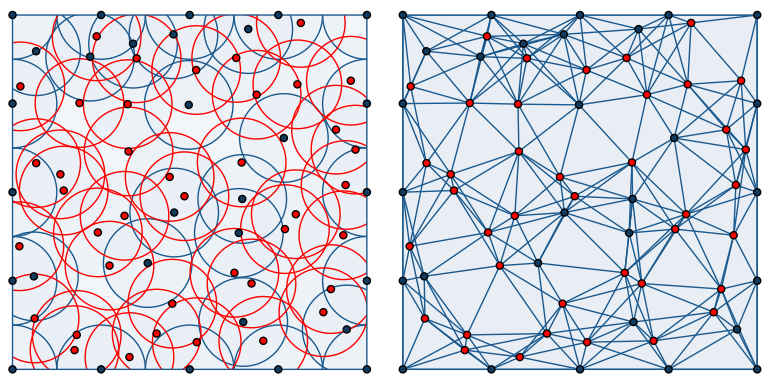

FiguRE 3. With the determinantal addition method with a Ginibre basis.

\subsubsection{Comparison}

To compare the methods efficiency, we count the number of vertices each method adds on average to reach complete coverage. The grid method being determinist, the number of added vertices is constant. We can see in Table 1 the mean number of added vertices on 1000 simulations for each method in different scenarios on a square of side $a=1$ with coverage radius $r=0.25$, and a Vietoris-Rips complex (graph approximation of the Cech complex). Scenarios are defined by the mean percentage of area covered initially.

The determinantal method provides the best results in all scenarios among the random methods by far. And it is the best method among all for the almost covered scenario. The grid method is more efficient for least covered scenarios, however it is not always practicable in a real life scenario where positions can not be defined with such precision, and any imprecision leads to a coverage hole.

\subsection{Reduction algorithm}

The next step of our algorithm is to run the coverage reduction algorithm from [45]. At this step, we remove some of the supernumerary vertices we just added in order to achieve a minimal result with a minimum number of added vertices. The reduction algorithm takes as input an abstract simplicial complex: here it is the Čech complex of the wireless network plus the added vertices, and a list of boundary vertices. Then the algorithm removes vertices from the simplicial complex without modifying its two Betti numbers. In [45], we show that the configuration reached by the algorithm is maximal: it can not be improved. For more information on the reduction algorithm we refer to [45]. We can see in Figure 4 an execution of the reduction algorithm on the intermediate configuration of Figure 3 Removed vertices are empty circles.
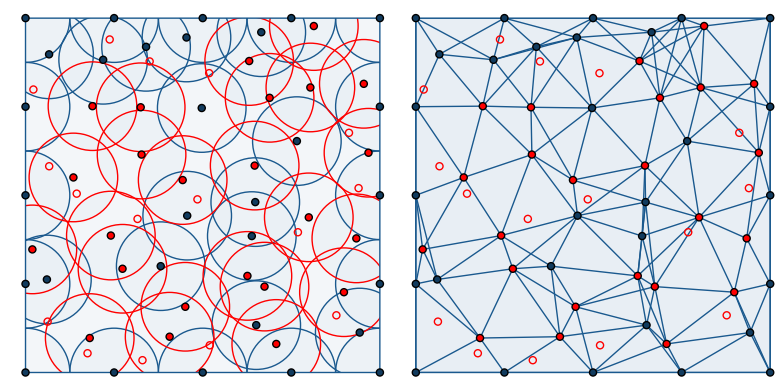

FIGURE 4. The reduction algorithm run on the determinantal method example.

\subsection{Performance comparison with a greedy algorithm}

We now compare the performance results of the whole disaster recovery algorithm to the most known coverage recovery algorithm: the greedy algorithm for the set cover problem. We compare the final number of added 


\begin{tabular}{|lcccc|}
\hline Percentage of area initially covered & $20 \%$ & $40 \%$ & $60 \%$ & $80 \%$ \\
\hline $\mathbb{E}\left(N_{a}\right)$ & & & & \\
\hline Grid method & 9.00 & 9.00 & 9.00 & 9.00 \\
\hline Uniform method & 32.51 & 29.34 & 24.64 & 15.63 \\
\hline Determinantal method & 16.00 & 14.62 & 12.36 & 7.79 \\
\hline $\mathbb{E}\left(N_{f}\right)$ & & & & \\
\hline Greedy algorithm & 3.69 & 3.30 & 2.84 & 1.83 \\
\hline Homology algorithm & 4.42 & 3.87 & 2.97 & 1.78 \\
\hline
\end{tabular}

TABLE 1. Mean number $\mathbb{E}\left(N_{a}\right)$ and mean final number $\mathbb{E}\left(N_{f}\right)$ of added vertices

\begin{tabular}{|lcccc|}
\hline Percentage of area initially covered & $20 \%$ & $40 \%$ & $60 \%$ & $80 \%$ \\
\hline $\mathbb{E}\left(\beta_{1}\right)$ & & & & \\
\hline Greedy algorithm & 0.68 & 0.65 & 0.45 & 0.35 \\
\hline Homology algorithm & 0.62 & 0.53 & 0.37 & 0.26 \\
\hline $\mathbb{P}\left(\beta_{1}=0\right)$ & & & & \\
\hline Greedy algorithm & $40.8 \%$ & $47.7 \%$ & $61.0 \%$ & $69.3 \%$ \\
\hline Homology algorithm & $50.9 \%$ & $58.1 \%$ & $67.9 \%$ & $75.3 \%$ \\
\hline
\end{tabular}

TABLE 2. Changes caused by the Gaussian perturbation

vertices. Results presented in Table 1 are simulated in the same conditions as in Section 3.2 The numbers of vertices added in the final state both tend to the number of vertices needed to cover the initially uncovered area. Nonetheless, we can see that our algorithm performs a little bit worse than the greedy algorithm in the less covered area scenarios because whole parts of the grid from the greedy algorithm are used. In compensation, our homology algorithm performs better in more covered scenarios.

We then evaluate the robustness of the algorithms when the real positioning does not strictly follow the theoretical one. In order to do this, we apply a Gaussian perturbation to each added vertex. The covariance matrix of the perturbation is given by $\Sigma=\sigma^{2} \mathrm{Id}$ with $\sigma^{2}=0.01$. We compute the mean number of holes, and the percentage of simulations in which no hole is created after the Gaussian perturbation. Results are shown in Table 2

Our homology algorithm performs better, there are less than $50 \%$ of simulations that create coverage holes. The greedy algorithm always creates more coverage holes in mean than our disaster recovery algorithm for the same vertices positions perturbation. Therefore our algorithm seems more fitted to the disaster recovery case when a recovery network is deployed in emergency both indoor, via Femtocells, and outdoor, via a trailer fleet, where exact GPS locations are not always available, and exact theoretical positioning is not always followed.

\section{Sublinearity of the MeAn Number of SEMI-INFinite BRANCHES OF GEOMETRIC RANDOM GRAPHS}

Contrarily to abstract graphs (as Erdös-Rényi graphs or planar maps) where the positions of vertices have no importance and where only the topology of the structure matters, the geometric random graphs are embedded into a state space. Their graph structures depend on the spatial positions of the vertices through local or global geometric rules, and not only through probabilistic procedures. The interest of the scientific community for these graphs has recently increased because of the huge variety of modern applications where they appear (Astronomy, Biology, Telecommunications...).

When each vertex of a given geometric random tree $\mathcal{T}$ built on a countable vertex set, has finite degree then $\mathcal{T}$ automatically contains at least one semi-infinite branch. Except this elementary result, describing the semi-infinite branches of $\mathcal{T}$ is not a trivial problem. An important step was taken by Howard and Newman 
in [30]. They develop a robust and efficient method (Proposition 2.8) ensuring that a geometric planar random tree $\mathcal{T}$ satisfies the two following statements :

- $[S 1]$ a.s. every semi-infinite branch of $\mathcal{T}$ starting at its root has an asymptotic direction;

- [S2] a.s. for every direction $\theta \in[0 ; 2 \pi)$, there is at least one semi-infinite branch of $\mathcal{T}$ starting at its root with asymptotic direction $\theta$.

As an obvious consequence, for such a tree, its number $\chi_{r}$ of semi-infinite branches starting at the root and crossing the sphere $S(O, r)$ with radius $r$ a.s. tends to infinity.

The main condition to get $[S 1]$ and $[S 2]$ is that the tree $\mathcal{T}$ has to be straight (see Section 2.3 of [30]). Roughly speaking, this means that the subtrees of $\mathcal{T}$ are becoming thinner when their roots are far away from the one of $\mathcal{T}$. In the last 15 years, the straight character has been established for many geometric random trees. Here we focus our attention on two examples of straight geometric random trees; the Radial Poisson Tree and the Euclidean First-Passage Percolation Tree. For these two models, our main result says the expectation of $\chi_{r}$ is sublinear w.r.t. $r$ :

$$
\lim _{r \rightarrow \infty} \mathbb{E}\left(\frac{\chi_{r}}{r}\right)=0 .
$$

This result means that among all the edges crossing the sphere $S(O, r)$, whose mean number is of order $r$, a very few number of them belong to semi-infinite branches. When the law of the geometric random tree is isotropic, the limit (4) is deduced from the following directional result. Let $\chi_{r}(\theta, c)$ be the number of semi-infinite branches (or geodesics) of the considered tree starting at the root and crossing the arc of $S(O, r)$ centered at $r e^{\mathbf{i} \theta}$ and with length $c$. Then,

$$
\lim _{r \rightarrow \infty} \mathbb{E}\left(\chi_{r}(\theta, c)\right)=0 .
$$

\subsection{Models and sublinearity results}

\subsubsection{Notation}

Let $O$ be the origin of $\mathbb{R}^{2}$ which is endowed with the Euclidean norm $\|\cdot\|$. We denote by $B(X, r)$ the open euclidean ball with center $X$ and radius $r$, and by $S(X, r)$ the corresponding sphere. The two dimensional real plan $\mathbb{R}^{2}$ and the set of complex numbers $\mathbb{C}$ are identified. Hence, according to the context, a point $X$ will be described by its cartesian coordinates $(X(1), X(2))$ or its Euclidean norm $\|X\|$ and its argument $\arg (X)$ which is the unique real number $\theta$ in $[0 ; 2 \pi)$ such that $X=\|X\| e^{\mathbf{i} \theta}$.

Both trees considered in the next sections are graphs with out-degree 1 (except for their roots). The outneighbor of any vertex $X$ will be denoted by $A(X)$ and called the ancestor of $X$. We will also say that $X$ is a child of $A(X)$.

Moreover, about these trees, we will keep the same notation that they are considered as random graphs with edges $\{X, A(X)\}$ or as subsets of $\mathbb{R}^{2}$ made up of segments $[X ; A(X)]$. This second interpretation will particularly be suitable for the study of their semi-infinite branches which are some (unbounded) sequences $\left(X_{n}\right)_{n \in \mathbb{N}}$ of points of $\mathcal{N}$ such that, for any $n, A\left(X_{n+1}\right)=X_{n}$. Such a branch admits $\theta \in[0 ; 2 \pi)$ as asymptotic direction if $X_{n} /\left\|X_{n}\right\|$ tends to $e^{\mathbf{i} \theta}$ with $n$.

\subsubsection{The Radial Poisson Tree}

Let us consider a homogeneous Poisson Point Process (PPP) $\mathcal{N}$ in $\mathbb{R}^{2}$ with intensity 1 . Let $\rho>0$ be a positive real number. Considering $\mathcal{N} \cap B(O, \rho)^{c}$ instead of $\mathcal{N}$, we can assume for this section that $\mathcal{N}$ has no point in $B(O, \rho)$. The Radial Poisson Tree $(\mathrm{RPT}) \mathcal{T}_{\rho}$ is a (directed) graph whose vertex set is given by $\mathcal{N} \cup\{O\}$. Let us define the ancestor $A(X)$ of any vertex $X \in \mathcal{N}$ as follows. First we set

$$
\operatorname{Cyl}(X, \rho)=([O ; X] \oplus B(O, \rho)) \cap B(O,\|X\|),
$$

where $\oplus$ denotes the Minkowski sum. If $\operatorname{Cyl}(X, \rho) \cap \mathcal{N}$ is empty then $A(X)=O$. Otherwise, $A(X)$ is the element of $\operatorname{Cyl}(X, \rho) \cap \mathcal{N}$ having the largest Euclidean norm:

$$
A(X)=\operatorname{argmax}\{\|Y\|, Y \in \operatorname{Cyl}(X, \rho) \cap \mathcal{N}\} .
$$


Hence, the set $\operatorname{Cyl}(X, \rho)^{*}=\operatorname{Cyl}(X, \rho) \backslash B(O,\|A(X)\|)$ avoids the PPP $\mathcal{N}$. See Figure 5

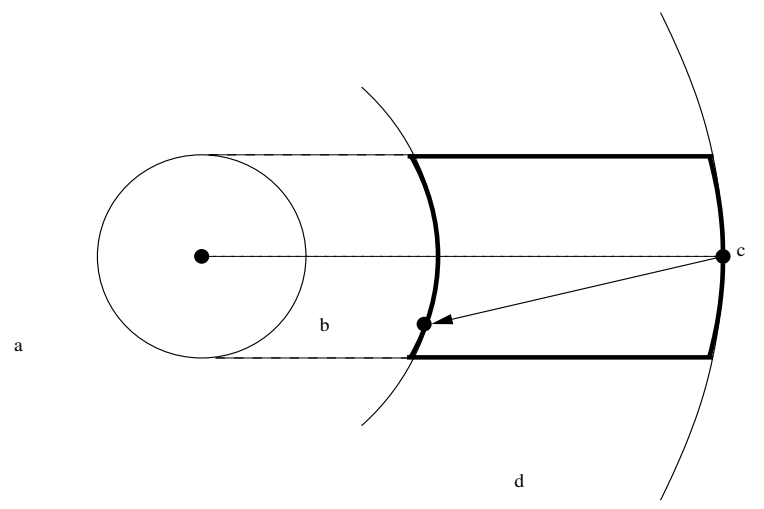

FiguRE 5. This picture represents the ancestor $A(X) \in \mathcal{N}$ of $X=(x, 0)$. The bold lines delimit the cylinder $\operatorname{Cyl}(X, \rho)^{*}$.

This construction ensures the a.s. uniqueness of the ancestor $A(X)$ of any $X \in \mathcal{N}$. This means that the RPT has no loop. Besides, $A(X)$ is closer than $X$ to the origin. Since the PPP $\mathcal{N}$ is locally finite, then any $X \in \mathcal{N}$ is linked to the origin by a finite number of edges. Moreover, it is not difficult to see that the number of children of any $X \in \mathcal{N} \cup\{O\}$ is a.s. finite but unbounded.

The main motivation to study this model comes from the fact it is closely related- in a sense which will be specified in Section 4.2 - to a directed forest introduced by Ferrari and its coauthors in [17].

Now, let us focus on the semi-infinite branches of the Radial Poisson Tree $\mathcal{T}_{\rho}$. Our first result states that the RPT is straight. Then, the method of Howard and Newman (Proposition 2.8 of [30]) applies: any semi-infinite branch of the RPT has an asymptotic direction and in each direction there is a semi-infinite branch.

Theorem 3. The Radial Poisson Tree $\mathcal{T}_{\rho}$ a.s. satisfies statements [S1] and [S2].

The number $\chi_{r}$ of semi-infinite branches of the RPT $\mathcal{T}_{\rho}$ at level $r$ is the number of intersection points of the sphere $S(O, r)$ with the semi-infinite branches of the RPT. Proposition 3 states that $\chi_{r}$ a.s. converges to infinity as $r \rightarrow \infty$. Thus, consider two real numbers $\theta \in[0 ; 2 \pi]$ and $c>0$. We denote by $\chi_{r}(\theta, c)$ the number of semi-infinite branches of the RPT crossing the arc of $S(O, r)$ centered at $r e^{\mathbf{i} \theta}$ and with length $c$.

Theorem 4. Let $\theta \in[0 ; 2 \pi]$ and $c>0$ be real numbers. Then,

$$
\lim _{r \rightarrow \infty} \mathbb{E}\left(\frac{\chi_{r}}{r}\right)=0 \text { and } \lim _{r \rightarrow \infty} \mathbb{E}\left(\chi_{r}(\theta, c)\right)=0
$$

Furthermore, the sequence $\left(\chi_{r}(\theta, c)\right)_{r>0}$ does not tend to 0 a.s.:

$$
\mathbb{P}\left(\limsup _{r \rightarrow \infty} \chi_{r}(\theta, c) \geq 1\right)=1 .
$$

\subsubsection{The Euclidean FPP Tree}

Let $\alpha>0$ be a positive real number. The Euclidean First-Passage Percolation Tree $\mathcal{T}_{\alpha}$ introduced and studied in [29,30], is a planar graph whose vertex set is given by a homogeneous Poisson Point Process (PPP) $\mathcal{N}$ in $\mathbb{R}^{2}$ with intensity 1 . Unlike the RPT whose graph structure is local, that of the Euclidean FPP Tree is global and results from a minimizing procedure.

Let $X, Y \in \mathcal{N}$. A path from $X$ to $Y$ is a finite sequence $\left(X_{1}, \ldots, X_{n}\right)$ of points of $\mathcal{N}$ such that $X_{1}=X$ and $X_{n}=Y$. To this path, the weight $\sum_{i=1}^{n-1}\left\|X_{i}-X_{i+1}\right\|^{\alpha}$ is associated. Then, the path minimizing this weight is 
called the geodesic from $X$ to $Y$ and denoted by $\gamma_{X, Y}$ :

$$
\gamma_{X, Y}=\operatorname{argmin}\left\{\sum_{i=1}^{n-1}\left\|X_{i}-X_{i+1}\right\|^{\alpha}, n \geq 2 \text { and }\left(X_{1}, \ldots, X_{n}\right) \text { is a path from } X \text { to } Y\right\} \text {. }
$$

By concavity of $x \mapsto x^{\alpha}$ for $0<\alpha \leq 1$, the geodesic from $X$ to $Y$ coincides with the straight line $[X ; Y]$. Since a.s. no three points of $\mathcal{N}$ are collinear, it is reduced to the trivial path $(X, Y)$. So, from now on, to get nontrivial geodesics, we assume $\alpha>1$.

Existence and uniqueness of the geodesic $\gamma_{X, Y}$ is a.s. ensured whenever $\alpha>1$ (Proposition 1.1 of [30]). Let $X^{O}$ be the closest point of $\mathcal{N}$ to the origin $O$. The Euclidean FPP Tree $\mathcal{T}_{\alpha}$ is defined as the collection $\left\{\gamma_{X^{O}, X}, X \in \mathcal{N}\right\}$. By uniqueness of geodesics, $\mathcal{T}_{\alpha}$ is a tree rooted at $X^{O}$.

Any vertex $X$ of $\mathcal{T}_{\alpha}$ a.s. has finite degree (Proposition 1.2 of $[30]$ ). The ancestor $A(X)$ of $X$ is, among all its neighbors, the one belonging to the geodesic $\gamma_{X}{ }^{O}, X$ (if $\left.X \neq X^{O}\right)$. The other ones are the children of $X$.

The straight character of the Euclidean FPP Tree is stated in Theorem 2.6 of [30] (for $\alpha>1$ ). It then follows:

Theorem 5 (Theorems 1.8 and 1.9 of [30]). For any $\alpha>1$, the Euclidean FPP Tree $\mathcal{T}_{\alpha}$ a.s. satisfies statements [S1] and $[S 2]$.

The definition of the number $\chi_{r}$ of semi-infinite geodesics of the Euclidean FPP Tree $\mathcal{T}_{\alpha}$ at level $r$ requires to be more precise than in Section 4.1.2. Indeed, the vertices of geodesics of $\mathcal{T}_{\alpha}$ are not sorted w.r.t. their Euclidean norms and these geodesics may cross many times any given sphere. So, let us consider the graph obtained from $\mathcal{T}_{\alpha}$ after deleting any geodesic $\left(X^{O}, X_{2}, \ldots, X_{n}\right)$ with $n \geq 2$ (except the endpoint $\left.X_{n}\right)$ such that the vertices $X^{O}, X_{2}, \ldots, X_{n-1}$ belong to the ball $B\left(X^{O}, r\right)$ but not $X_{n}$. Then, $\chi_{r}$ counts the infinite connected components of this graph. Now, let $\theta \in[0 ; 2 \pi]$ and $c>0$. The random integer $\chi_{r}(\theta, c)$ denotes the number of these infinite connected components whose geodesics linking them to $X^{O}$ in $\mathcal{T}_{\alpha}$ cross the sphere $S\left(X^{O}, r\right)$ on the arc centered at $X^{O}+r e^{\mathbf{i} \theta}$ and with length $c$.

Theorem 6. Let $\theta \in[0 ; 2 \pi]$ and $c>0$ be real numbers. Assume $\alpha \geq 2$. Then,

$$
\lim _{r \rightarrow \infty} \mathbb{E}\left(\frac{\chi_{r}}{r}\right)=0 \text { and } \lim _{r \rightarrow \infty} \mathbb{E}\left(\chi_{r}(\theta, c)\right)=0 .
$$

Furthermore, the sequence $\left(\chi_{r}(\theta, c)\right)_{r>0}$ does not tend to 0 a.s.:

$$
\mathbb{P}\left(\limsup _{r \rightarrow \infty} \chi_{r}(\theta, c) \geq 1\right)=1 .
$$

The hypothesis $\alpha \geq 2$ is added so that the Euclidean FPP Tree $\mathcal{T}_{\alpha}$ satisfies the non-crossing property (Lemma 5 of $[29]$.

\subsection{Sketch of the proofs}

In this section, we use the generic notation $\mathcal{T}$ to refer to the RPT $\mathcal{T}_{\rho}$ and to the Euclidean FPP Tree $\mathcal{T}_{\alpha}$.

First of all, the isotropic property of $\mathcal{T}$ allows to reduce the study to one given direction $\theta$ :

$$
\mathbb{E}\left(\chi_{r}\right)=r \mathbb{E}\left(\chi_{r}(\theta, 2 \pi)\right) .
$$

So, our goal is to show that the expectation of $\chi_{r}(\theta, 2 \pi)$ tends to 0 as $r$ tends to infinity (we will take $\theta=0$ ). The scheme of the proof can be divided into four steps.

STEP 1: The first step consists in approximating locally (i.e. around the point $r e^{\mathbf{i} \theta}$ ) and in distribution the tree $\mathcal{T}$ by a directed forest, say $\mathcal{F}$, with direction $-e^{\mathbf{i} \theta}$. The directed forest $\mathcal{F}$ chosen to approximate the RPT has been introduced by Ferrari et al. 17. This forest is given by the collection of coalescing one-dimensional 
random walks with uniform jumps in a bounded interval (with radius $\rho$ ) and starting at the point of a homogeneous Poisson Point Process in $\mathbb{R}^{2}$. Its graph structure is based on local rules. Conversely, the directed forest chosen to approximate the Euclidean FPP Tree is the collection of coalescing infinite geodesics with direction $-e^{\mathbf{i} \theta}$. Consequently, the proofs of Step 1 for the RPT and for the Euclidean FPP Tree will be radically different.

STEP 2: The goal of the second step is to prove that the directed forest $\mathcal{F}$ (with direction $-e^{\mathbf{i} \theta}$ ) has a.s. only one topological end. To do it, we first state that all the branches (or geodesics) eventually coalesce (in the sense $\left.-e^{\mathbf{i} \theta}\right)$. Thus, we deduce that $\mathcal{F}$ does not contain any bi-infinite branch (or geodesic).

STEP 3: Combining results of the two previous steps, we get that $\chi_{r}(\theta, 2 \pi)$ tends to 0 in probability, as $r$ tends to infinity. Let us roughly describe the underlying idea. The event $\chi_{r}(\theta, 2 \pi) \geq 1$ implies the existence of a very long branch (or geodesic) of the tree $\mathcal{T}$ crossing the arc of the sphere $S(O, r)$ centered at $r e^{\mathbf{i} \theta}$ and with length $c$. Thanks to Step 1, the probability of this latest event is close to the one of the event corresponding to the existence of a branch (or geodesic) of the directed forest $\mathcal{F}$ crossing the segment centered at $O$, with length $c$ and orthogonal to $-e^{\mathbf{i} \theta}$, which is very long in the sense $e^{\mathbf{i} \theta}$. Now, thanks to Step 2, this should not happen.

STEP 4: In this last step, we exhibit a uniform (on $r$ ) moment condition for $\chi_{r}(\theta, 2 \pi)$ to strengthen its convergence to 0 in the $L^{1}$ sense.

\section{RANDOM WALKS ON RANDOM DELAUNAY TRIANGULATIONS}

Let us begin by describing the model. Given an infinite locally finite set of points $\xi \subset \mathbb{R}^{d}$, the Voronoi cell with nucleus $x \in \xi$ is defined by:

$$
\operatorname{Vor}_{\xi}(x)=\left\{y \in \mathbb{R}^{d}:\|y-x\| \leq\left\|y-x^{\prime}\right\|, \forall x^{\prime} \in \xi\right\}
$$

The collection of the Voronoi cells $\left\{\operatorname{Vor}_{\xi}(x), x \in \xi\right\}$ is the Voronoi tiling of $\xi$. If the convex hull of $\xi$ is $\mathbb{R}^{d}$, it forms a partition of $\mathbb{R}^{d}$ into convex polyhedra. In the sequel, we consider Voronoi tilings generated by point processes in $\mathbb{R}^{d}$ which have very different clustering or repulsiveness properties. When the underlying point process is a Poisson point process, the corresponding model is known as Poisson-Voronoi tessellation. It was introduced in 1953 by Meijering [38] to study the formation of crystals. Actually, the Voronoi tiling of a locally set of points $\xi$ provides a natural division of the space when there exists some competition for resources. Indeed, the Voronoi cell $\operatorname{Vor}_{\xi}(x)$ can be thought as the territory of the nucleus $x$ when nuclei expand their respective territories at the same speed or as the set of the points of $\mathbb{R}^{d}$ that prefer to be attached at $x$ rather than at an other point of $\xi$ by proximity. The model thus found applications in various fields such that geography [5, 6], biology [23], astronomy [40] or telecommunications 2, 19,20].

Since Voronoi tilings generated by point processes arise naturally in various concrete situations, it is important to study random walks which move, at each step, from the nucleus of a Voronoi cell to the nucleus of a neighboring cell. In other words, it is to study nearest-neighbor random walks on the dual graph of the Voronoi tiling of $\xi$ : the Delaunay triangulation $\mathrm{DT}(\xi)$. This graph has $\xi$ as vertex set and there is an edge between $x$ and $y$ in $\mathrm{DT}(\xi)$ if and only if $\operatorname{Vor}_{\xi}(x)$ and $\operatorname{Vor}_{\xi}(y)$ share a $(d-1)$-dimensional face.

In the sequel, we will present recurrence and transience results for simple random walks on Delaunay triangulations generated by point processes in $\mathbb{R}^{d}$ and study their behavior at the diffusive scale from an averaged (on the environments) and an almost-sure point of view. Namely, we will state an annealed and a quenched invariance principle for such random walks. These results can be compared with their counterpart for other models of random walks in random environments already studied in the literature. To be precise, the transience of random walks on supercritical percolation clusters in $\mathbb{Z}^{d}, d \geq 3$, is shown in 25 . Invariance principle for random walks on supercritical percolation clusters or other random perturbations of the grid $\mathbb{Z}^{d}$ can be found e.g. in [11] (annealed) and [3, 4, 35, 36, 44] (quenched). Random walks on complete graphs generated by point 
processes with jump probabilities decreasing with the distance between points are studied in [8] (recurrence and transience), [14] (annealed invariance principle) and [9] (quenched invariance principle).

Recurrence and transience. The question of the recurrence or the transience of random walks on Delaunay triangulations generated by point processes in $\mathbb{R}^{d}$ was investigated in 41 . in wich the following theorem is proved.

Theorem 7 (see [41, Theorems 1 and 2]). Under assumptions on the underlying point process satisfied in particular by homogeneous Poisson point processes, Matérn Cluster processes and Matérn Hardcore processes, for a.a. realization of $\xi$, the simple nearest-neighbor random walk on $\mathrm{DT}(\xi)$ is:

- null recurrent if $d=2$,

- transient if $d \geq 3$.

The precise assumptions on the point process can be found in [41, §1.1] and other examples of random geometric graphs for which the conclusions of the theorem above are valid can be found in paragraph $\S 1.2$ of that paper. Let us note that recurrence and transience results for random walks on Poisson-Delaunay triangulations were first announced in the unpublished manuscript [1]. Their proofs relied on a deviation result for the socalled stabbing number of $\mathrm{DT}(\xi)$ and the reference then cited is unfortunately unavailable. The deviation result for the stabbing number has been proved since then in [39] but the method developed in [41] avoids the use of such a strong estimate and is thus more tractable. The recurrence in dimension 2 is obtained thanks to a criterion (namely [41, Criterion 4]) which is close in spirit to the Nash-Williams criterion. Transience in higher dimensions is obtainded as follows. We show that, for a.a. realization of $\xi$, there exists a rough-embedding (see $33, \S 2.6]$ ) from a realization of a supercritical percolation cluster in $\mathbb{Z}^{d}$ to DT $(\xi)$. Since, it is known that a.a. realization of the supercritical percolation cluster is transient for $d \geq 3$ (see 25]), the result then follows from 33, Theorem 2.17].

Annealed invariance principle. In order to study the behavior of the random walk rescaled at the diffusive scale in average on the environments, we need to assume that 0 is in a.a. realization of the point process. To make it precise, we consider random set of points $\xi^{0}$ distributed according to the Palm measure $\mathcal{P}_{0}$ associated with the point process (see $[10, \S 4.4 .2]$ for a definition). It gives mass 1 to the set $\mathscr{N}_{0}$ of all infinite locally finite subsets of $\mathbb{R}^{d}$ containing 0 .

For $\xi^{0} \in \mathscr{N}_{0}$, we denote by $\left(\mathbf{X}_{t}^{\xi^{0}}\right)_{t \geq 0}$ the continuous-time random walk on $\xi^{0}$ starting at 0 and with infinitesimal generator given by:

$$
\mathcal{L}^{\xi^{0}} f(x)=\sum_{y \in \xi^{0}} \mathbf{1}_{y \sim x \text { in } \mathrm{DT}\left(\xi^{0}\right)}(f(y)-f(x)), x \in \xi^{0} .
$$

We denote by $P_{0}^{\xi^{0}}$ the law of $\left(\mathbf{X}_{t}^{\xi^{0}}\right)_{t \geq 0}$ and by $E_{0}^{\xi^{0}}$ the corresponding expectation.

Theorem 8 (see 42 , Theorem 1]). If $\xi^{0}$ is distributed according to the Palm measure $\mathcal{P}_{0}$ associated with a suitable point process in $\mathbb{R}^{d},\left(\mathbf{X}_{t}^{\xi^{0}}\right)_{t \geq 0}$ satisfies an annealed invariance principle.

More precisely, the rescaled process $\left(\varepsilon \mathbf{X}_{\varepsilon^{-2} t}^{\xi^{0}}\right)_{t \geq 0}$ converges weakly in $\mathcal{P}_{0}$-probability to a nondegenerate Brownian motion with diffusion coefficient given by:

$$
\sigma^{2}=\lim _{t \rightarrow \infty} \frac{1}{t} \int_{\mathscr{N}_{0}} E_{0}^{\xi^{0}}\left[\left(\mathbf{X}_{t}^{\xi^{0}} \cdot e_{1}\right)^{2}\right] \mathcal{P}_{0}\left(\mathrm{~d} \xi^{0}\right)>0,
$$

where $e_{1}$ is the first vector of the standard basis of $\mathbb{R}^{d}$.

The precise assumptions on the point process are listed in [42, §1.2] and are satisfied for exemple by Poisson point processes, Matérn Cluster processes and Matérn Hardcore processes. In [42, §2], the use of the process of the environment seen from the particle makes possible to rewrite $\left(\mathbf{X}_{t}^{\xi^{0}}\right)_{t \geq 0}$ as an additive functional of a Markov process under the annealed measure $\mathbf{P}[\cdot]=\int P_{0}^{\xi^{0}}[\cdot] \mathcal{P}_{0}\left(\mathrm{~d} \xi^{0}\right)$. An application of $[11$, Theorem 2.2] then gives the 
convergence to a Brownian motion. The main difficulty is to prove that its diffusion coefficient $\sigma^{2}$ is positive. As in 11, 14, this is done by comparing $\sigma^{2}$ with effective conductances of suitable electrical networks. These conductances can be finally bounded from below, after a discretisation of the space, using technics and results form percolation theory (see $[42, \S 3]$ ).

Quenched invariance principle. For $\xi$ an infinite locally finite subset of $\mathbb{R}^{d}$, let us consider the continuoustime random walk $\left(\mathbf{X}_{t}^{\xi}\right)_{t \geq 0}$ on $\xi$ with infinitesimal generator given by 12 . For $x \in \xi$, we denote by $P_{x}^{\xi}$ the law of this random walk with initial condition $\mathbf{X}_{0}^{\xi}=x$.

Theorem 9 (see [43, Theorem 1]). Assume that $\xi$ is distributed according to a simple, stationary, isotropic point process with law $\mathcal{P}$ a.s. in general position, satisfying the assumptions given in [43, \$1.1]. Then, $\left(\mathbf{X}_{t}^{\xi}\right)_{t \geq 0}$ satisfies a quenched invariance principle.

More precisely, for $\mathcal{P}$-a.e. $\xi$, for all $x \in \xi$, under $P_{x}^{\xi}$, the rescaled process $\left(\varepsilon \mathbf{X}_{\varepsilon^{-2} t}^{\xi}\right)_{t \geq 0}$ converges in law as $\varepsilon$ tends to 0 to a nondegenerate Brownian motion with diffusion coefficient $\sigma^{2}$ which is positive and does not depend on $\xi$.

As for the previous results, the assumptions of Theorem 9 are satisfied by Poisson point processes, Matérn hardcore processes and Matérn cluster processes.

Thanks to 9 . Lemma B.2], Theorem 9 follows from its counterpart for the random walk sarting at 0 on $\xi^{0}$ generated by the Palm measure $\mathcal{P}_{0}$ associated with the stationary point process (see [43. Theorem 30]). This last is proved using the corrector method: it is shown that the random walk behaves like a martingale up to a correction which is negligible at the diffusive scale.

Theorem 10. Under the assumptions of Theorem 9, there exists a so-called corrector $\chi: \mathscr{N}_{0} \times \mathbb{R}^{d} \longmapsto \mathbb{R}^{d}$ such that:

(1) $\varphi\left(\xi^{0}, x\right)=x-\chi\left(\xi^{0}, x\right)$ is harmonic at 0 for $\mathcal{P}_{0}-$ a.e. $\xi^{0}$, i.e.:

$$
\sum_{x \in \xi^{0}} \mathbf{1}_{x \sim 0 \text { in } \mathrm{DT}\left(\xi^{0}\right)}\left\|\varphi\left(\xi^{0}, x\right)\right\|<\infty \text { and } \mathcal{L}^{\xi^{0}} \varphi\left(\xi^{0}, 0\right)=0 \text { for } \mathcal{P}_{0}-\text { a.e. } \xi^{0}
$$

(2) $\chi$ is a.s. sublinear:

$$
\frac{\max _{x \in \xi^{0} \cap[-n, n]^{d}}\left\|\chi\left(\xi^{0}, x\right)\right\|}{n} \frac{\underset{\mathcal{P}_{0}-a . s .}{\longrightarrow}}{n \rightarrow \infty} .
$$

The arguments to deduce the version of Theorem 9 for Palm measures from Theorem 10 are rather standard (see e.g. [9 §3.3.1], [4, p. 1340-1341] or [3, §6.1 and §6.2]). The existence of an harmonic deformation of $\mathrm{DT}\left(\xi^{0}\right)$ which corresponds to the existence of the corrector was obtained in 18 but its sublinearity follows from their proofs only in dimension 2 . The existence of the corrector is obtained in [43, §2] by using the Weyl decomposition of $L^{2}(\mu)$ where $\mu$ is the measure on $\mathscr{N}_{0} \times \mathbb{R}^{d}$ defined by:

$$
\int u \mathrm{~d} \mu:=\int_{\mathscr{N}_{0}} \sum_{x \in \xi^{0}} \mathbf{1}_{0 \sim x} \operatorname{in} \mathrm{DT}\left(\xi^{0}\right) u\left(\xi^{0}, x\right) \mathcal{P}_{0}\left(\mathrm{~d} \xi^{0}\right) .
$$

In a first time, the sublinearity of the corrector is obtained in an infinite subgraph $\mathcal{G}_{\infty}\left(\xi^{0}\right)$ of the Delaunay triangulation $\mathrm{DT}\left(\xi^{0}\right)$ which has good regularity properties. The vertices of this subgraph have bounded degrees, its volume grows regularly and it is possible to bound from below its isoperimetric profile. The method developed in [4] is then adapted to prove that:

$$
\frac{\max _{x \in \mathcal{G}_{\infty}\left(\xi^{0}\right) \cap[-n, n]^{d}}\left\|\chi\left(\xi^{0}, x\right)\right\|}{n} \frac{\underset{\mathcal{P}_{0}-a . s .}{n \rightarrow \infty}}{n} 0 .
$$


Let us note that this method was also successfully used in the context of random walks on complete graphs generated by point processes with jump probabilities decreasing with the distance between points in $[9]$. Since the connected components of $\mathrm{DT}\left(\xi^{0}\right) \backslash \mathcal{G}_{\infty}\left(\xi^{0}\right)$ are a.s. finite, the sublinearity of the corrector can finally be extended to the full Delaunay triangulation by harmonicity.

\section{REFERENCES}

[1] L. Addario-Berry and A. Sarkar. The simple random walk on a random Voronoi tiling. Available at http://www.dms. umontreal.ca/ addario/papers/srwd.pdf, 2005.

[2] F. Baccelli, F. and B. Blaszczyszyn. Stochastic Geometry and Wireless Networks, Volume 1: Theory, Volume 2: Applications. NOW Publishers, Foundations and Trends in Networking, 2009.

[3] N. Berger and M. Biskup. Quenched invariance principle for simple random walk on percolation clusters. Probab. Theory Related Fields, 137: 83-120, 2007.

[4] M. Biskup and T. M. Prescott. Functional CLT for random walk among bounded random conductances. Electron. J. Probab., 12: $1323-1348,2007$.

[5] B. N. Boots. Some models of the random subdivision of space. Geografiska Annaler, 55B: 34-48, 1973.

[6] B. N. Boots. Some observations on the structure of socioeconomic cellular networks. The Canadian Geographer, 19: 107-120, 1975 .

[7] R. M. Burton and M. S. Keane. Density and uniqueness in percolation. Comm. Math. Phys., 121:501-505, 1989.

[8] P. Caputo, A. Faggionato and A. Gaudillière. Recurrence and transience for long range reversible random walks on a random point process. Electron. J. Probab., 14: 2580-2616, 2009.

[9] P. Caputo, A. Faggionato and T. Prescott. Invariance principle for Mott variable range hopping and other walks on point processes. Ann. Inst. H. Poincaré Probab. Statist., 49: 654-697, 2013.

[10] S. N. Chiu, D. Stoyan, W. S. Kendall and J. Mecke. Stochastic geometry and its applications. Wiley Series in Probability and Mathematical Statistics: Applied Probability and Statistics, third edition, John Wiley \& Sons Ltd., Chichester, 2013.

[11] A. De Masi, P. A. Ferrari, S. Goldstein and W. D. Wick. An invariance principle for reversible Markov processes. Applications to random motions in random environments. J. Statist. Phys., 55: 787-855, 1989.

[12] L. Decreusefond, I. Flint, and A. Vergne. Efficient simulation of the Ginibre process. J. of Appl. Probab., $52,2015$.

[13] M. Deijfen. Asymptotic shape in a continuum growth model. Adv. in Appl. Probab., 35:303-318, 2003.

[14] A. Faggionato, H. Schulz-Baldes and D. Spehner. Mott law as lower bound for a random walk in a random environment. Comm. Math. Phys., 263: 21-64, 2006.

[15] Q. Fang, J. Gao, and L. Guibas. Locating and bypassing routing holes in sensor networks. INFOCOM 2004. Twenty-third Annual Joint Conference of the IEEE Computer and Communications Societies, 4: 2458-2468, 2004.

[16] P. A. Ferrari, L. R. G. Fontes, and Xian-Yuan Wu. Two-dimensional Poisson trees converge to the Brownian web. Ann. Inst. H. Poincaré Probab. Statist., 41(5):851-858, 2005.

[17] P. A. Ferrari, C. Landim, and H. Thorisson. Poisson trees, succession lines and coalescing random walks. Ann. Inst. H. Poincaré Probab. Statist., 40:141-152, 2004. Probabilités et Statistiques.

[18] P. A. Ferrari R. M. Grisi and P. Groisman. Harmonic deformation of Delaunay triangulations. Stochastic Process. Appl., 122: 2185-2210, 2012.

[19] S. G. Foss and S. A. Zuyev. On a Voronoi aggregative process related to a bivariate Poisson process. Adv. in Appl. Probab., 28: 965-981, 1996.

[20] C. Gloaguen, F. Voss and V. Schmidt. Parametric distributions of connection lengths for the efficient analysis of fixed access network. Ann. of Telecom., 66: 103-118, 2011.

[21] J.-B. Gouéré. Subcritical regimes in the Poisson Boolean model of continuum percolation. Ann. Probab., 36:1209-1220, 2008.

[22] J.-B. Gouéré and R. Marchand. Continuous first-passage percolation and continuous greedy paths model: linear growth. Ann. Appl. Probab., 18:2300-2319, 2008.

[23] M. Gerstein, J. Tsai and M. Levitt. The volume of atoms on the protein surface : Calculated from simulation, using Voronoi polyhedra. J. of Mol. Biol., 249: 955-966, 1995.

[24] R. Ghrist and A. Muhammad. Coverage and hole-detection in sensor networks via homology. Proceedings of the 4th international symposium on Information processing in sensor networks, IPSN '05, Piscataway, NJ, USA, IEEE Press., 2005.

[25] G. R. Grimmett, H. Kesten and Y. Zhang. Random walk on the infinite cluster of the percolation model. Probab. Theory Related Fields, 96: 33-44, 1993.

[26] G. Grimmett. Percolation, volume 321 of Grundlehren der Mathematischen Wissenschaften [Fundamental Principles of Mathematical Sciences]. Springer-Verlag, Berlin, second edition, 1999.

[27] P. Hall. On continuum percolation. Ann. Probab., 13:1250-1266, 1985.

[28] A. Hatcher. Algebraic Topology. Cambridge University Press, 2002.

[29] C. D. Howard and C. M. Newman. Euclidean models of first-passage percolation. Probab. Theory Related Fields, 108(2):153$170,1997$. 
[30] C. D. Howard and C. M. Newman. Geodesics and spanning trees for Euclidean first-passage percolation. Ann. Probab., $29(2): 577-623,2001$.

[31] H. Kesten. Aspects of first passage percolation. In École d'été de probabilités de Saint-Flour, XIV-1984, volume 1180 of Lecture Notes in Math., 125-264. Springer, Berlin, 1986.

[32] C. Licea and C. M. Newman. Geodesics in two-dimensional first-passage percolation. Ann. Probab., 24(1):399-410, 1996.

[33] R. Lyons and Y. Peres. Probability on Trees and Networks. Download available from http://mypage.iu.edu/ rdlyons/prbtree/prbtree.html, 2014.

[34] J. B. Martin. Linear growth for greedy lattice animals. Stochastic Process. Appl., 98:43-66, 2002.

[35] P. Mathieu, P. and A. Piatnitski. Quenched invariance principles for random walks on percolation clusters. Proc. R. Soc. Lond. Ser. A Math. Phys. Eng. Sci., 463: 2287-2307, 2007.

[36] P. Mathieu. Quenched invariance principles for random walks with random conductances. J. Stat. Phys., 130: 1025-1046, 2008.

[37] R. Meester and R. Roy. Continuum percolation, volume 119 of Cambridge Tracts in Mathematics. Cambridge University Press, Cambridge, 1996.

[38] J. L. Meijering. Interface area, edge length, and number of vertices in crystal aggregates with random nucleation. Philips Res. Rep., 8, 1953.

[39] L. P. R. Pimentel and R. Rossignol. Greedy polyominoes and first-passage times on random Voronoi tilings. Electron. J. Probab., 17: 12-31, 2012.

[40] M. Ramella, W. Boschin, D. Fadda and M. Nonino. Finding galaxy clusters using Voronoi tessellations. Astronomy and Astrophysics, 368: 776-786, 2001.

[41] A. Rousselle. Recurrence or transience of random walks on random graphs generated by point processes in $\mathbb{R}^{d}$. Preprint available at http://arxiv.org/abs/1305.4878, 2013.

[42] A. Rousselle. Annealed invariance principle for random walks on random graphs generated by point processes in $\mathbb{R}^{d}$. Preprint available at https://hal.archives-ouvertes.fr/hal-01079900, 2014.

[43] A. Rousselle. Quenched invariance principle for random walks on Delaunay triangulations. Preprint available at http://arxiv.org/abs/1412.5033, 2014.

[44] V. Sidoravicius and A. S. Sznitman. Quenched invariance principles for walks on clusters of percolation or among random conductances. Probab. Theory Related Fields, 129: 219-244, 2004.

[45] A. Vergne, L. Decreusefond, and P. Martins. Reduction algorithm for simplicial complexes. INFOCOM, 2013 Proceedings IEEE, 475-479, 2013.

[46] C. Zhang, Y. Zhang, and Y. Fang. Detecting coverage boundary nodes in wireless sensor networks. Networking, Sensing and Control, 2006. ICNSC '06. Proceedings of the 2006 IEEE International Conference, 868-873, 2006. 